\title{
EchoGéo
}

30 | 2014

Varia

\section{Variations sur la ville}

Jean-Louis Chaléard

\section{(2) OpenEdition}

Journals

Electronic version

URL: https://journals.openedition.org/echogeo/14094

DOI: 10.4000/echogeo.14094

ISSN: 1963-1197

\section{Publisher}

Pôle de recherche pour l'organisation et la diffusion de l'information géographique (CNRS UMR 8586)

\section{Electronic reference}

Jean-Louis Chaléard, "Variations sur la ville", EchoGéo [Online], 30 | 2014, Online since 27 January 2015, connection on 31 July 2021. URL: http://journals.openedition.org/echogeo/14094 ; DOI: https:// doi.org/10.4000/echogeo.14094

This text was automatically generated on 31 July 2021.

EchoGéo est mis à disposition selon les termes de la licence Creative Commons Attribution - Pas d'Utilisation Commerciale - Pas de Modification 4.0 International (CC BY-NC-ND) 


\title{
Variations sur la ville
}

\author{
Jean-Louis Chaléard
}

1 Par définition un numéro Varia ne s'organise pas autour d'un thème unique. Pourtant la nouvelle livraison d'EchoGéo est placée sous le signe de la ville. Les différents articles de la rubrique Sur le champ portent sur des questions urbaines ou sur le périurbain. Sans doute cette concordance reflète-t-elle l'évolution d'un monde où la population citadine dépasse la population rurale depuis 2007, d'après les données de l'ONU. Au-delà des incertitudes des chiffres et des définitions variables de la ville selon les pays et les recensements, le monde est bien devenu majoritairement urbain. Et ce numéro d'EchoGéo en est le reflet.

Centrés sur des questions urbaines, les cinq articles de la rubrique Sur le champ sont cependant fort divers, témoignant de la multiplicité des situations et des approches. Ils sont divers d'abord par les lieux, abordant des villes d'Europe, d'Amérique latine, d'Afrique. Ensuite, par les méthodes, qui associent en proportions variables analyses statistiques et approches qualitatives, et recourent à des sources variées, des recensements nationaux aux images satellites. En outre, les études se situent à différentes échelles : d'une analyse dans un espace national (la Pologne), à une microgéographie (un hôpital à Ouagadougou), en passant par les cadres urbains (Mexico ou Port-au-Prince). Enfin, les thèmes révèlent quelques préoccupations majeures des chercheurs sur la ville, en résonnance avec la multiplicité des questions qui s'y posent : l'accès au foncier (à propos des villes polonaises), la santé (au Burkina Faso), les déchets (Port-au-Prince), la pauvreté (Mexico), les espaces verts (Abidjan)...

Dans le contexte des bouleversements opérés en Europe de l'est, après la fin de l'ère socialiste, l'article de C. Bouloc et M. Górczyńska explique la manière dont l'habitat s'est restructuré en Pologne, avec la réintroduction de la propriété privée, et analyse les conflits sociaux et spatiaux qui résultent des modes d'accession à la propriété. A. Nikiema nous introduit, de façon originale, dans l'espace intime du CHU de Ouagadougou pour comprendre les éléments qui composent le paysage thérapeutique, ses conséquences sur les malades et sa signification sur le système de soins, et pour présenter les éléments d'un modèle d'organisation. La question des déchets tient une place de plus en plus grande dans les villes aujourd'hui. Le texte de R. Popescu, 
M. Durand et R. D'Ercole en aborde un aspect original : la gestion des déchets postcatastrophe, prenant l'exemple de Port-au-Prince, douloureusement frappée par le terrible tremblement de terre de 2010. L'article montre que la principale méthode d'élimination des déchets est la relégation, mais il relève aussi la complexité des situations, la mise en concurrence des différentes urgences face à la catastrophe et in fine, l'intérêt d'une planification préventive. A. Ribardière et J.-F. Valette nous proposent, à partir d'un examen des données du recensement de 2010 au Mexique, une analyse des formes socio-spatiales de la pauvreté urbaine à Mexico. L'article met en évidence la diversité sociale des colonies populaires, nom générique donné aux quartiers populaires souvent auto-construits, nés dans l'illégalité foncière. Cette diversité interroge la spécificité des formes de peuplement et renvoie tant aux trajectoires de consolidation qu'à la dimension politique du processus de régularisation. Sur un autre plan, N. Sako et G. Beltrando s'intéressent au parc national du Banco, situé à l'intérieur du district d'Abidjan, à la périphérie de la ville, menacé par l'avancée des constructions urbaines. L'approche s'appuie à la fois sur des analyses d'images satellites, de relevés de terrains et d'enquêtes auprès des populations. Il est remarquable de constater que malgré l'encerclement progressif du parc par la ville, sa couverture végétale reste à dominante forestière. Cette relative préservation du parc national conduit à méditer sur les relations entre la ville et la campagne ou la ville et la "nature ", plus complexes qu'on ne l'a écrit parfois, dans un monde où un nombre croissant de citadins est à la recherche d'espaces verts et où la préservation de ces espaces en périphérie des métropoles est une des préoccupations majeures des autorités municipales et/ou nationales dans la plupart des grandes métropoles.

Le dossier de la rubrique Sur le métier s'intéresse aussi à la ville. B. Michelon appartient au Groupe Huit, bureau qui a réalisé de nombreuses études d'aménagement du territoire et d'urbanisme. Certains de ses membres interviennent dans le master 2 «Pratique du développement » (proposé par les Universités Paris 1 Panthéon-Sorbonne et Denis Diderot- Paris 7). À partir de la présentation des travaux en ateliers d'étudiants (sur le développement de Kinshasa et sur le modèle de l'éco-quartier dans les pays du Sud), B. Michelon montre l'intérêt pour les uns et les autres de ces formations, comment les professionnels initient les étudiants et comment ceux-ci en retour avec leurs savoirs et leur sensibilité de jeunes géographes contribuent à la réflexion sur la fabrication de la ville aujourd'hui.

5 Les rubriques Sur l'écrit et Sur le vif sont d'un tout autre ordre, illustrant des visages différents de la géographie. Dans la première, $S$. Blanchard nous propose une réflexion sur «l'intersectionalité » qui rend compte de l'imbrication des discriminations de classe, de genre et de «race ", à partir de la lecture croisée de deux ouvrages, celui d'E. N. Glenn sur la construction racialisée de l'emploi domestique au XX $\mathrm{X}^{\mathrm{e}}$ siècle et celui de $\mathrm{C}$. Avril sur les aides à domicile en France au début du XXI ${ }^{e}$ siècle. La lecture croisée des travaux de ces deux sociologues paraît riche de promesse pour une géographie sociale des migrations. Au-delà, elle montre l'intérêt de la confrontation entre des recherches françaises et américaines, et entre des sciences sociales voisines (la sociologie pouvant ici inspirer la géographie).

6 La rubrique Sur le vif est l'occasion pour G. Cullier de Labadie et M. Redon de réfléchir sur les enjeux des découpages territoriaux. Rappelant, après la visite de mai 2014 en Carladez du Prince de Monaco, les liens historiques entre la principauté et son ancien 
fief, ils s'interrogent de façon plus large sur ce qui donne sa légitimité à un découpage territorial, dans le contexte actuel de remise en cause de la carte des régions en France.

7 Il est impossible de terminer cet éditorial sans évoquer les effroyables attentats commis début janvier 2015 à Paris au siège de Charlie-Hebdo, au magasin Hyper Cacher de la Porte de Vincennes et à Montrouge. Si ces évènements dépassent largement le cadre d'une revue de sciences humaines et sociales, ils nous rappellent combien il est important de défendre la liberté d'expression, pour nous chercheurs aussi, sans laquelle nous ne pourrions exercer notre réflexion ni construire nos savoirs, et la nécessité de diffuser les acquis de la recherche, qui participent au progrès de la connaissance, condition nécessaire de la lutte contre l'intolérance. C'est ce à quoi nous nous attelons. 